\title{
WGMs on-chip design for interdisciplinary studies
}

\section{Preecha P. Yupapin}

Advanced Studies Center, Department of Physics, Faculty of Science, King Mongkut's Institute of Technology Ladkrabang (KMITL), Bangkok 10520, Thailand

\section{Email address:}

kypreech@kmitl.ac.th(P. P. Yupapin)

\section{To cite this article:}

Preecha P. Yupapin. WGMs on-Chip Design for Interdisciplinary Studies. Science Journal of Education. Vol. 1, No. 1, 2013 , pp.1-5. doi: 10.11648/j.sjedu.20130101.11

\begin{abstract}
Light in a PANDA ring resonator system for coated and uncoated materials have been proposed and discussed. The input waves (lights) can be in the forms of soliton, Gaussian pulses, photon or matter wave. The trapped electron motion around the PANDA ring can also be generated, in which the matter wave concept is formed within the PANDA ring waveguide. From the results, we found that the whispering gallery mode (WGM) can be generated due to coupling effects of the two nonlinear side rings, which have shown many interesting results and aspects. We found that four behaviors of light, for instance, fast, slow, stopping and storing can be manipulated and seen simultaneously by PANDA ring planar waveguide, which can be fabricated and tested on-chip, which is also suitable to implement in the laboratory.
\end{abstract}

Keywords: Micro-Optics, Optical Device, Integrated Optical System, Whispering Gallery Mode, PANDA Ring

\section{Introduction}

Whispering gallery modes (WGMs) of waves in nature such as electromagnetic wave, sound wave and matter wave have shown the interesting results which can be useful for fundamental studies and applications in optoelectronics and nano-electronics, especially, after the announcement of Nobel Prize 2012 in Physics on the WGMs [1, 2], where the authors have confirmed that atoms can be trapped (stopped) by using the WGM in a microsphere. However, there are two more kinds of devices that can be used to trap light beams (atoms), the use of microcavity arrays performed by Yanik and Fan [3], and a nonlinear microring resonator by Yupapin and Pornsuwancharoen [4] for stopping light. Recently, Ang and Ngo [5] have also done experiment to slowing light in microresonators using a microring system.

In this article, a new design of microring resonator device is proposed which can be used to generate four forms of light simultaneously on a chip, whereas the storing and harvesting of trapped atoms/molecules can also be available. The proposed device is made up of silica and InGaAsP/InP with linear optical add-drop filter incorporating two nonlinear micro/nano rings on both sides of the center ring (modified add-drop filter). This particular configuration is known as a "PANDA" ring resonator [6] as shown in Fig.1. Light pulse, for instance, Gaussian, bright and dark solitons is fed into the system through different ports such as add port and through port. By using the practical device parameters, the simulation results are obtained using the Optiwave and MATLAB programs. Results obtained by both analytical and numerical methods show that many applications can be exploited. In application, when the practical device parameters are used, then such device can be fabricated and implemented in the near future. The use of the system in laboratory is also possible.

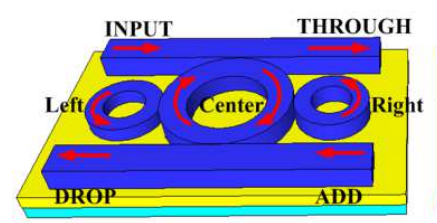

(A)Uncoated

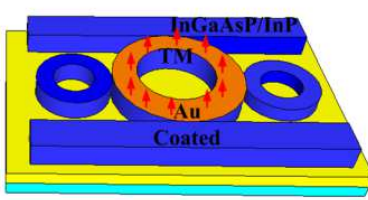

(B) Coated
Figure 1. (A) a conventional PANDA ring planar wave guide was named and designed

\section{Methodology}

The whispering gallery mode result is obtained using the Optiwave program as shown in Fig. 2. The ring material is InGaAsP/InP, where the device parameters are given in figure caption. By using the MATLAB program, the whispering gallery modes of four state of light i.e. fast, slow, stopping and storing can be generated and controlled simultaneously on-chip as shown in Fig. 3. The storing stage can be seen easily, while the stopping condition can be observed as the following conditions are satisfied: (i) the 
center signal is lost in time between fast and slow signals or (ii) there is no movement among trapped particles or molecules i.e. the exchange of angular momentum introduces the conservation of angular momentum, where the combination of scattering and gradient forces is balanced under the adiabatic process.

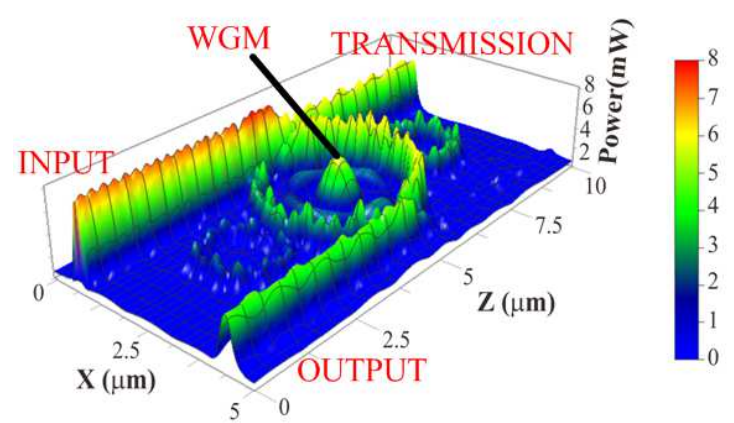

Figure 2. Result of whispering gallery mode of light within a PANDA ring waveguide InGaAsP/InP, $R_{1}=R_{2}=0.775 \mu \mathrm{m}, \quad R_{a d}=1.565$, Aeff $=0.3 \mu \mathrm{m}^{2}$, neff $=3.14, n_{2}=1.3 \times 10^{-13} \mathrm{~cm}^{2} / W, \kappa_{1}=\kappa_{2}=\kappa_{3}=\kappa_{4}=0.5, \quad \gamma=0.01, \lambda_{0}$ $=1,550 \mathrm{~nm}$
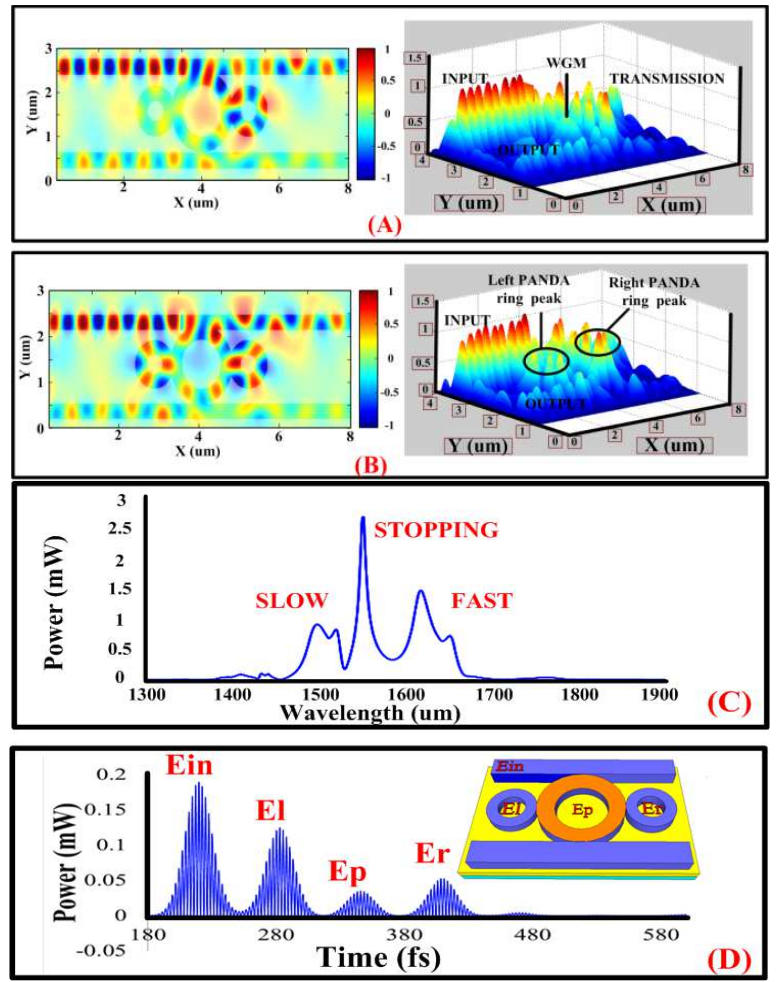

Figure 3. Stopping and storing light simultaneously detected using a $P A N D A$ ring, where $(A)$ Center ring, (B) Side rings, (C) Center peak and side peaks, (D) Fast (2nd peak) and slow (4th peak) light detected at drop port with time interval of $150 \mathrm{fs}$

The stopping light in term of signal condition can be easily performed using the whispering gallery mode concept, where the fast and slow light can be used as the upper and lower time frames or upper side and lower side peak signals for the storing light at the center as shown in Fig. 3, where in this case the movement (modulated signals) longer than 150 fs, i.e. ms, ns, ps is observed (stopped). The input pulse is a
Gaussian pulse with pulse width of $100 \mathrm{fs}$, where the fast and slow time interval is known, however, the whispering gallery modes can be seen only under the resonant condition.

The use of light trapping probe for atom/molecule trapping and transportation (dynamically trapping) can be formed with wide range of applications. In this case the modulated signal is required to switch off the whispering gallery mode power via the add port, where atoms/molecules at the device center can be trapped and transported along the wave guide by the surface plasmon tweezers as shown in Fig. 4. The dynamic tweezers are generated by a PANDA ring, whereas in practice, particle angular momentum can be introduced by a metal coating material on the waveguide surface or combining the external modulation via the add port, which can be used to trap and transport atom/molecule to the required destination when the gradient force is greater than the scattering force along the waveguide. The use of such concept for new type solar cells is as shown in Fig. 5, where free electrons from the depletion region can be trapped and transported(injected) to the metal contact faster than the conventional device, the solar cells efficiency can be increased by 5 times to the conventional one.

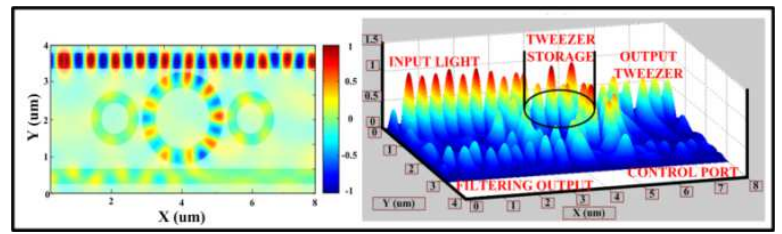

Figure 4. Dynamic tweezers are generated by a PANDA ring and transmitted via a Through port for atoms/molecules harvesting and transportation

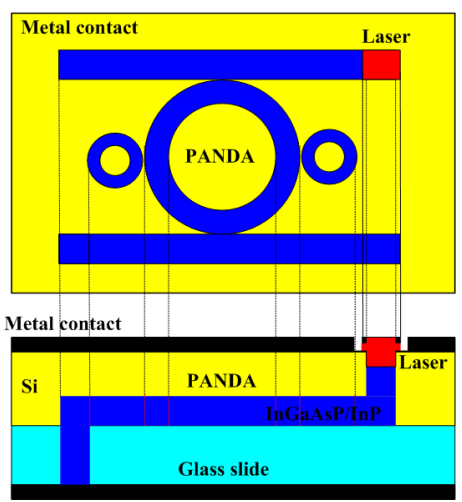

(a) Design structure

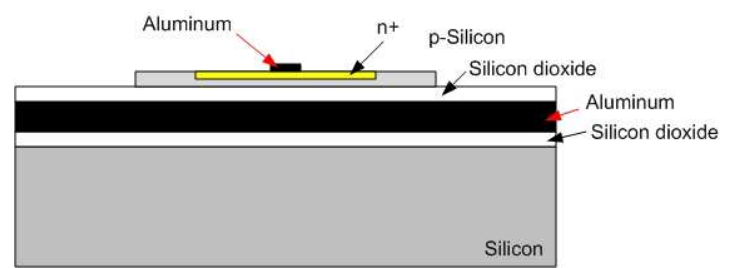

(b) Fabrication structure

Figure 5. New type solar cells using a PANDA and embedded particle accelerator (Optical trapping and transportation) [8, 9] 
From the above reasons, we found that the WGM of light can be easily formed by a microring resonator [7] or nonlinear coupling effects to the center ring of a PANDA ring [8]. In Fig. 6, the WGM and leaky modes are generated by a PANDA ring which can be formed in the same ways for large scale motion. Thus, the use of WGM for large scale motion can be useful, for instance, the WGM between the Earth and Moon orbital motion, where in this case the matter wave(wave-particle duality) can be formed by the Moon orbital motion around the Earth, which can be useful for Earth disaster investigation and prediction. Another application is the atomic modulation can be formed by using the WGM of light in a PANDA ring as shown in Fig. 7, where the atoms are dancing and atomic radio can be performed and realized by the external modulation to the trapped atoms.

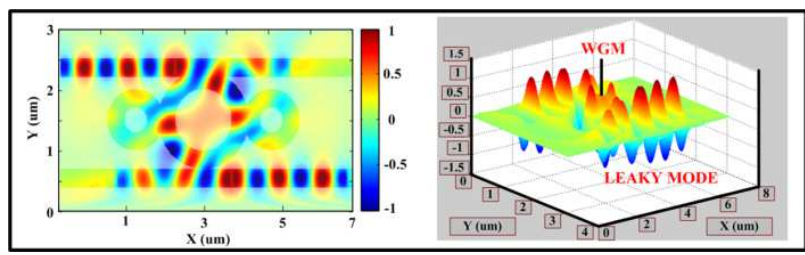

Figure 6. WGMs and leaky modes generated by a PANDA ring

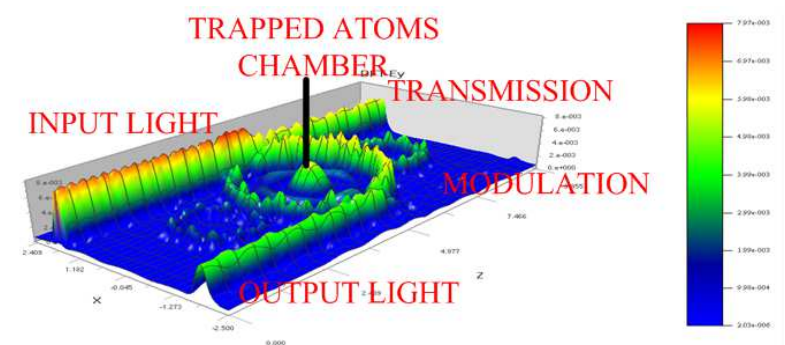

Figure 7. Schematic diagram of trapping and modulating atom by light

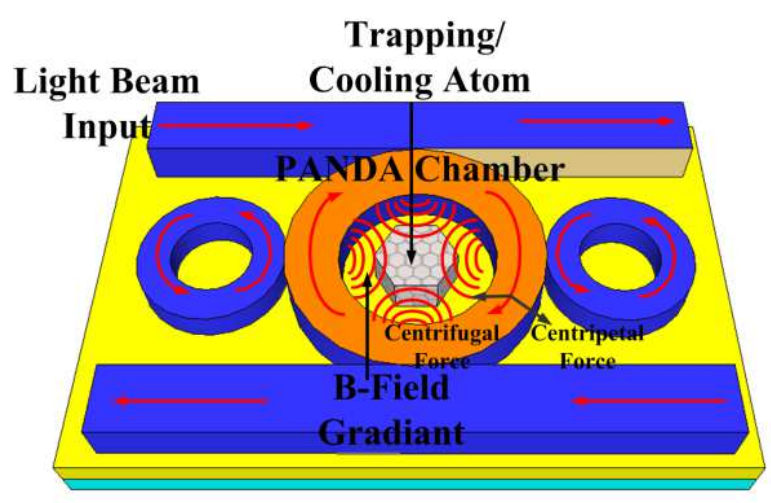

Figure 8. PANDA chamber for particle/photon trapping and storage under (i) centripetal and centrifugal forces, (ii) WGMs

To perform the experiment that atoms can interact with light one of the techniques uses a standard magneto-optic trap (MOT)[11], where analysis of the cold collision measurements performed in a high-gradient magneto-optical trap with a few trapped Cs atoms. The net result is a cloud of cold, confined cesium atoms inside a vacuum chamber, providing a convenient initial condition for optical-lattice experiments. While in the optical lattice, the trapping fields are extinguished so that they do not interfere with the atomic dynamics in the lattice.

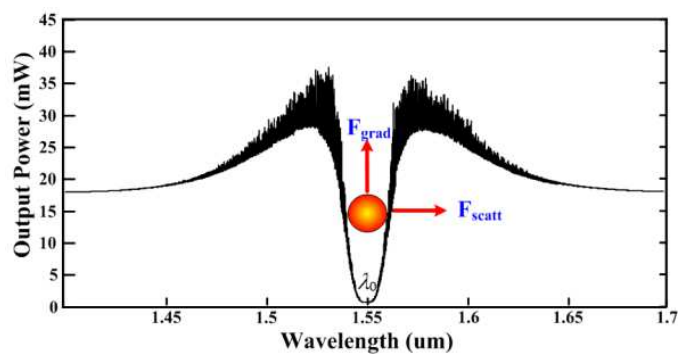

Figure 9. Particle (photon) is trapped by a potential well within a PANDA ring waveguide, where $F_{\text {grad }}$ : Gradient force, $F_{\text {scatt }}$ : Scattering force

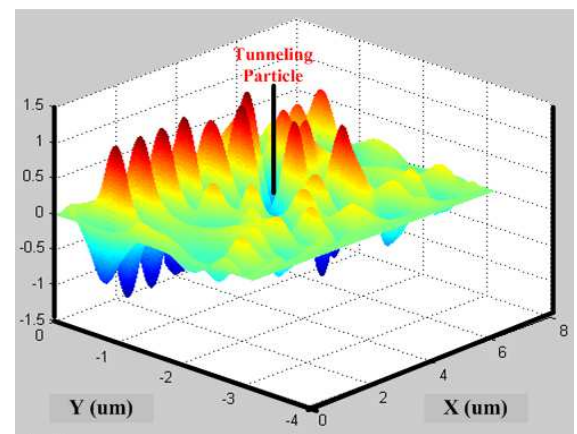

Figure 10. Dynamic tunneling particles/photons, where $X$ and $Y$ : two dimension scale

In our FDTD simulation, the perfectly matched layer (PML) absorbing boundary conditions have been applied by Berenger [12] and Yee scheme [13], which absorbs the electromagnetic wave without any reflection at the computational boundary. A 100 fs-Gaussian pulse modulated by a $200 \mathrm{THz}$ carrier is exited. The vertical waveguide thickness and material composition is accounted by computing the effective refractive index $n_{\text {eff }}$ for the fundamental mode at $\lambda=1.55 \mu \mathrm{m}$. In the vertical direction, each waveguide structure is $0.45 \mu \mathrm{m}$ thick, vertical core thicknesses of $0.3 \mu \mathrm{m}$ to $0.5 \mu \mathrm{m}, n_{\text {eff }}$ is between 3.2 to 3.4 , in which the parameters are obtained by using the practical material parameters of InGaAsP/InP. Therefore, the waveguide core $n=3.14$ is bordered on each side by air $n=1$. The parameters for add-drop optical multiplexer and both nanorings on the left and right hand sides of the PANDA ring are set at $\mathrm{R}_{\mathrm{l}}=\mathrm{R}_{\mathrm{r}}=0.75 \mu \mathrm{m}$ and radius of the center ring is $\mathrm{Rad}=1.5 \mu \mathrm{m}$. The coupling coefficient ratios are $\kappa_{1}=\kappa_{4}=0.5, \quad \kappa_{2}=\kappa_{1}=0.4$, effective core area of the waveguides is $A_{\text {eff }}=0.25 \mu \mathrm{m}^{2}$, and waveguide loss coefficient is $\alpha=0.1 \mathrm{~dB} / \mathrm{mm}$. When gold material is coupled as a function of frequency by coating on the waveguide as shown in Fig. 1, the free carrier concentration of gold is $N \approx 6 \times 10^{22} \mathrm{~cm}^{-3}$ and the plasma frequency is $\omega_{p} / 2 \pi \approx 2 \times 10^{3}$ 


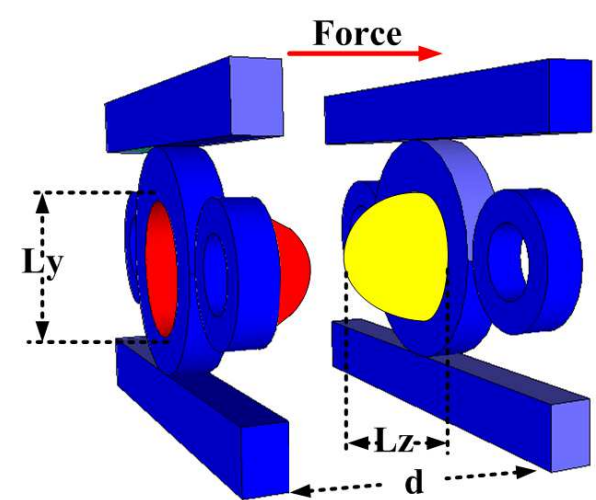

Barrier Gap

(a)

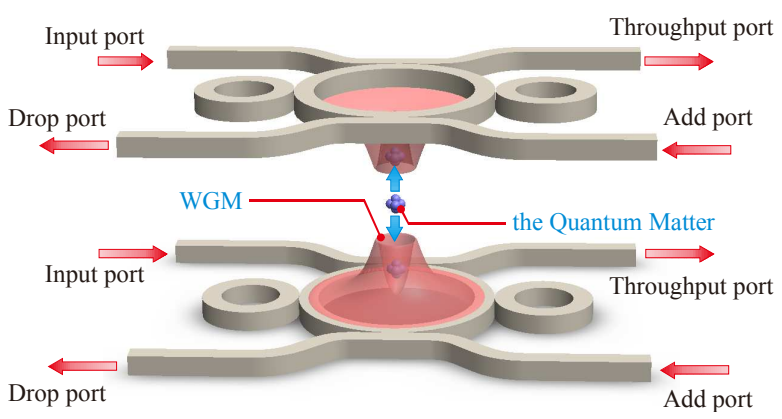

(b)

Figure 11. (a) WGMs switching control for particle/photon confinement (storage) and harvesting, where $L_{y}, L_{z}:$ WGMs dimension, $d$ : Device(Barrier) Gap, (b)shows the technique that can be used to form the quantum matter by particle tunneling and injecting into the sample material

In this article, the whispering gallery modes can be generated by trapping particle/photons within a PANDA rings as shown in Fig. 8, where the tunneling particles/photons can be generated when the particle energy is greater than the trapping potential as shown in Fig. 9. By using the MATLAB program, the whispering gallery modes of tunneling particles/photons can be generated and controlled on-chip as shown in Fig. 10. The tunneling particles/photons can be confined by the WGMs at the center, which can be used as particle source for various applications as shown in Fig. 11.

The proposed system can be used for applications such as atom/molecule transportation, everlasting atom/molecule investigation, atomic/molecular storage for quantum gate or computer application, storm search and navigator sensors, micro plasma source, 3-D flat panel device and large cooling area (volume), etc. In this work one of them is demonstrated. Under the stopping and storing condition, where the system is considered as the storage unit, in which atom or molecule can be trapped by the whispering gallery light beam at the center, where the trapped atoms/molecules can be modulated by the external modulated signals. Forms of light in a PANDA ring resonator with or without coated material can be manipulated, and it can be observed that the two nonlinear side rings have shown the interesting results and aspects. The input light can be in the forms of soliton or Gaussian pulses. The use of photon or matter wave as input is also possible. Particularly, an interesting aspect can also be formed by using the trapped electron, in which the matter wave concept can arise within the PANDA ring waveguide.

\section{Conclusion}

In conclusion, we found that four behaviors of light, for instance, fast, slow, stopping and storing can be manipulated and seen simultaneously by using the PANDA ring planar waveguide, which can be fabricated and tested on-chip, which can be performed in laboratory for teaching applications. The expected output light can be in the forms of surface plasmon, potential wells, leaky modes, whispering gallery modes, matter wave and photons (particles). The use of nonlinear Schrodinger equation can also be available, where in this case the propagation of light being treated as a particle (photon) within the PANDA ring, in which the tunneling effects of particles can be performed and investigated.

\section{References}

[1] Wineland, D.J., Bollinger, J.J., Itano, W.M. \&Prestage, J.D. Angular momentum of trapped atomic particles. JOSA B, 2, 1721-1730(1985).

[2] Knight, J.C. et al, Characterizing whispering-gallery modes in microspheres by direct observation of the optical standing-wave pattern in the near field. Opt. Lett., 21, 698-670(1996).

[3] Yanik, M.F. \& Fan, S. Stopping and storing light coherently. Phys. Rev. Lett., 92, 083901-3(2004).

[4] Yupapin, P.P. \&Pornsuwancharoen, N. Proposed nonlinear microring resonator arrangement for stopping and storing light. IEEE Photon. Techn.Lett., 21,404-406(2009).

[5] Ang, T.Y.L. \& Ngo, N.Q. Tunable flat-band slow light via contra-propagating cavity modes in twin coupled microresonators. JOSA B, 29, 924-933(2012).

[6] Uomwech, K., Sarapat, K. \&Yupapin, P.P. Dynamic modulated Gaussian pulse propagation within the double PANDA ring resonator system. Microw.\& Opt. Techn.Lett.,52, 1818-1821(2010).

[7] Kamoldilok S. \&Yupapin, P.P. Nanoheat source generated by leaky light mode within a nano-waveguide for small electrical power generator. Ener.Conver.\&Manag.64, 23-27(2012).

[8] Thammawongsa, N., Tunsiri, S., Jalil, M.A., Ali, J. \&Yupapin, P.P. Storing and harvesting atoms/molecules On-Chip: Challenges and applications. J. Biosensors \& Bioelectronics, 3, e114-115(2012).

[9] Srithanachai, I., Ueamanapong, S., Niemcharoen, S. \&Yupapin, P.P. Novel design of solar cell efficiency improvement using an embedded electron accelerator on-chip. Opt. Exp., 20, 12640-12648(2012). 
[10] Thammawongsa, N., Moonfangklang, N., Mitatha, S. \&Yupapin, P.P. Novel nano-antenna system design using photonics spin in a panda ring resonator. PIER L, 3, 75-87(2012).

[11] Ueberholz, B., Kuhr, S., Frese, D., Gomer, V. \&Meschede, $D$. Cold collisions in a high-gradient magneto-optical trap. $J$. Phys. B: At. Mol. Opt. Phys., 35, 4899-4914(2002).
[12] Berenger, J.P. Perfectly matched layer for the FDTD solution of wave structure interaction problem. IEEE Trans. Antennas Propagation, 44, 110-118(1996).

[13] Yee, K.S. Numerical solution of initial boundary value problems involving Maxwell's equations in isotropic media. IEEE Trans. Antennas Propagation, 14, 302-307 (1966). 